\title{
TENDÊNCIAS EVOLUTIVAS DE FAMÍLIAS PRODUTORAS DE CUMARINAS EM ANGIOSPERMAE
}

\section{Claudia Valeria Campos Ribeiro* e Maria Auxiliadora Coelho Kaplan}

Núcleo de Pesquisa de Produtos Naturais, Universidade Federal do Rio de Janeiro, CCS, Bloco H, Cidade Universitária, 21945-970 Rio de Janeiro - RJ

Recebido em 29/3/01; aceito em 9/11/01

\begin{abstract}
EVOLUTIONARY TENDENCY OF COUMARIN-BEARING FAMILIES IN ANGIOSPERMAE. Coumarins are special metabolites well distributed in the Angiospermae, either in Monocotyledoneae or Dicotyledoneae. Simple coumarins, the most widespread type, is found in all coumarin-producing families, such as: Apiaceae, Rutaceae, Asteraceae, Fabaceae, Oleaceae, Moraceae e Thymelaeaceae. The other types, linear- and angular furanocoumarins, linear- and angular pyranocoumarins, lignocoumarins, bis- and triscoumarins, are of more restricted circumscription. Among the families with occurrence numbers $(\mathrm{NO})>100$, the more advanced ones are specialized in the production of only one or two coumarin types, while the primitive families are very well diversified in types. Calculations of percentual numbers of occurrence (\%NO) show relevant meaning of coumarin-types in the taxonomic positioning of the producing taxa.
\end{abstract}

Keywords: coumarin; chemotaxonomy; evolution.

\section{INTRODUÇÃO}

Os estudos sobre sistemática e evolução de plantas, atualmente, estão bem fundamentados na existência de gradientes químicos de afinidade entre os grupos vegetais ${ }^{1}$. Gradientes químicos, podem ser compreendidos com base na correlação entre parâmetros químicos e morfológicos de avanço evolutivo do grupo vegetal em estudo ${ }^{2}$.

As cumarinas constituem uma classe química, sendo o primeiro representante isolado por Vogel, em 1820, da espécie Coumarona odorata $^{3}$. Esses metabólitos estão presentes em diferentes partes das plantas tanto nas raízes como nas flores e frutos e podem estar distribuídas em diferentes famílias de Angiospermae como Apiaceae, Rutaceae, Asteraceae nas quais são encontradas com ampla ocorrência. Também estão presentes em Fabaceae, Oleaceae, Moraceae e Thymeleaceae, entre outras, onde suas ocorrências são menores. Dentre os táxons que biossintetizam cumarinas contam espécies de hábitos bastante diversificados, como árvores, arbustos e ervas.

\section{PARTE EXPERIMENTAL}

O estudo quimiotaxonômico utilizando as cumarinas de Angiospermae foi iniciado através de levantamento dos dados da literatura. As informações sobre a ocorrência de cumarinas foram obtidas no Chemical Abstracts (Subject Index) de 1907 a 2000.

Posterior consulta aos artigos originais forneceu indicações completas sobre as cumarinas estudadas.

De posse dessas informações foram determinados vários parâmetros taxonômicos: Número de Ocorrência (NO) que expressa a capacidade da planta em produzir cumarinas; Número Percentual de Ocorrências (NO\%) que fornece o grau de relevância da ocorrência de cumarinas para um táxon em questão ${ }^{4,5}$. Número de Tipos (NT) de estruturas moleculares para cumarinas que expressa a capacidade da planta em modificar o esqueleto básico das cumarinas. O Índice de Sporne (IS), usado como indicador de tendências evolutivas morfológicas, quantifica características evolutivas morfológicas de famílias de Angiospermae ${ }^{6}$.

*e-mail: claudia@nppn.ufrj.br

\section{RESULTADOS E DISCUSSÃO}

O estudo da distribuição de cumarinas em táxons pertencentes a Angiospermae revela perfis característicos de ocorrência. Apesar da ampla distribuição das cumarinas, na classe, pode ser observado que a sua diversificação fica restrita a algumas famílias. A tendência de acúmulo de diferentes tipos desses metabólitos é bem clara para as poucas ordens como Araliales, Rutales, Urticales, Thymeleales e Fabales. Alguns táxons como Asterales, Oleales e outras, embora sejam grandes produtores desses metabólitos especializaram-se na produção de apenas alguns tipos ${ }^{7}$ (Tabela 1).

Para este trabalho foram selecionados oitos tipos de cumarinas: cumarinas simples (Cs), furanocumarinas lineares ( $\mathrm{Fcl}$ ), furanocumarinas angulares ( $\mathrm{Fca})$, piranocumarinas lineares $(\mathrm{Pcl})$, piranocumarinas angulares (Pca), lignocumarinas (Lc), biscumarinas (Bc) e triscumarinas (Tc) (Figura 1).

As trinta seis ordens apresentadas na Tabela 1 mostram os números de ocorrências (NO) por ordem, para esses diferentes tipos de cumarinas produzidas. As ordens Asterales, Caryophyllales, Sapindales, Lamiales, Malvales, Dipsacales, Geraniales, Capparales, Orchidales, Myrtales, Magnoliales produzem um número razoável de cumarinas, porém de apenas dois tipos: sempre cumarinas simples e um outro tipo qualquer. Por outro lado, Araliales, Rutales, Fabales, Urticales, Thymeleales, Gentianales são ordens bastante diversificadas produzindo de quatro a sete tipos de cumarinas (Tabela 1).

A Tabela 2 mostra as famílias de diferentes ordens produtoras de cumarinas com número de ocorrência maior do que vinte, além do número percentual de ocorrências de cumarinas para cada família dentro da ordem e o número de tipos para a família. Dos resultados apresentados pode ser observado que existem famílias cuja ocorrência de cumarinas significa aproximadamente cem por cento da ordem. Araliales é uma ordem formada por duas famílias: Apiaceae que além de ser a maior produtora de cumarinas com NO\% igual à 99,78 é a mais diversificada, contendo 5 tipos. Araliaceae apresenta $0,22 \%$ dessa produção metabólica e possui um único tipo. A segunda ordem em ocorrência de cumarinas é Rutales formada por cinco famílias produtoras desses metabólitos, na qual, Rutaceae apresenta uma grande superioridade tanto em ocorrência quanto em diversificação em tipos. Essas duas famílias, Apiaceae e Rutaceae, merecem destaque já que as 
Tabela 1. Números de ocorrências referentes às ordens de Angiospermae e aos tipos de cumarinas produzidas

\begin{tabular}{|c|c|c|c|c|c|c|c|c|c|}
\hline \multirow[t]{3}{*}{ Ordens } & \multicolumn{9}{|c|}{ NOs } \\
\hline & \multicolumn{9}{|c|}{ Tipos de cumarinas } \\
\hline & Total & $\mathrm{Cs}$ & $\mathrm{Fcl}$ & Fca & $\mathrm{Pcl}$ & Pca & $\mathrm{Lc}$ & $\mathrm{Bc}$ & $\mathrm{Tc}$ \\
\hline Araliales & 3048 & 1000 & 1449 & 321 & 73 & 205 & & & \\
\hline Rutales & 1724 & 881 & 580 & 25 & 147 & 39 & & 52 & \\
\hline Asterales & 830 & 819 & 11 & & & & & & \\
\hline Fabales & 245 & 190 & 32 & 14 & 7 & 2 & & & \\
\hline Oleales & 149 & 149 & & & & & & & \\
\hline Urticales & 139 & 47 & 73 & 10 & 9 & & & & \\
\hline Thymelaeales & 121 & 64 & 2 & 3 & 2 & & 3 & 44 & 3 \\
\hline Gentianales & 91 & 88 & 1 & 1 & & 1 & & & \\
\hline Solanales & 85 & 78 & 6 & & & & & 1 & \\
\hline Rosales & 69 & 65 & 2 & 2 & & & & & \\
\hline Saxifragales & 61 & 61 & & & & & & & \\
\hline Euphorbiales & 47 & 39 & & & & & 6 & 2 & \\
\hline Caryophyllales & 46 & 44 & 2 & & & & & & \\
\hline Sapindales & 44 & 39 & & & & & 5 & & \\
\hline Ericales & 35 & 35 & & & & & & & \\
\hline Lamiales & 24 & 16 & 8 & & & & & & \\
\hline Malvales & 22 & 21 & 1 & & & & & & \\
\hline Ranunculales & 21 & 21 & & & & & & & \\
\hline Ebenales & 16 & 16 & & & & & & & \\
\hline Polygalales & 16 & 16 & & & & & & & \\
\hline Dipsacales & 15 & 14 & 1 & & & & & & \\
\hline Balsaminales & 14 & 14 & & & & & & & \\
\hline Laurales & 14 & 14 & & & & & & & \\
\hline Violales & 12 & 12 & & & & & & & \\
\hline Poales & 11 & 11 & & & & & & & \\
\hline Geraniales & 9 & 7 & 2 & & & & & & \\
\hline Capparales & 9 & 5 & & & & & 4 & & \\
\hline Orchidales & 9 & 8 & 1 & & & & & & \\
\hline Myrtales & 9 & 8 & & & 1 & & & & \\
\hline Scrophulariales & 7 & 7 & & & & & & & \\
\hline Magnoliales & 7 & 6 & 1 & & & & & & \\
\hline Fagales & 6 & 4 & 1 & & 1 & & & & \\
\hline Liliales & 6 & 6 & & & & & & & \\
\hline Goodeniales & 5 & 5 & & & & & & & \\
\hline Cyperales & 4 & 2 & 1 & 1 & & & & & \\
\hline Juncales & 2 & 2 & & & & & & & \\
\hline Pittosporales & 1 & 1 & & & & & & & \\
\hline Campanulales & 1 & 1 & & & & & & & \\
\hline Buxales & 1 & 1 & & & & & & & \\
\hline Cornales & 1 & 1 & & & & & & & \\
\hline Aristolochiales & 1 & 1 & & & & & & & \\
\hline Annonales & 1 & 1 & & & & & & & \\
\hline Tamaricales & 1 & 1 & & & & & & & \\
\hline Theales & 1 & 1 & & & & & & & \\
\hline Loasales & 1 & 1 & & & & & & & \\
\hline Vitidales & 1 & 1 & & & & & & & \\
\hline Zingiberales & 1 & 1 & & & & & & & \\
\hline
\end{tabular}

Legenda: Cs (cumarina simples), Fcl (furanocumarina linear), Fca (furanocumarina angular), Pcl (piranocumarina linear), Pca (pirnocumrina angular), Lc (lignocumarina), Bc (bis-cumarina), Tc (tris-cumarina).

suas cumarinas podem ser consideradas verdadeiros marcadores quimiotaxonômicos. A ocorrência de cumarinas em Asterales é bastante significativa, porém cabe a observação que as cumarinas de Asteraceae são praticamente de um único tipo, cumarinas simples. Thymeleaceae distingue-se, por ser uma família que mais diversifica em tipos cumarínicos (7 entre total de 8). Fabaceae, é outra grande produtora de diferentes tipos estruturais (5). Em Urticales, a família responsável pela ocorrência mais expressiva desses metabólitos é a família Moraceae, onde podem ser encontrados também diferentes tipos de cumarinas (4). Oleales é uma ordem formada por apenas uma família Oleaceae e as cumarinas produzidas nesse táxon são apenas de um único tipo, cumarinas simples. 
<smiles>O=c1ccc2ccc(O)cc2o1</smiles>

Umbelliferona

Cumarina simples (Cs)<smiles>COc1cc2ccc(=O)oc2c2ccoc12</smiles>

Sphondina

Furanocumarina angular (Fca)<smiles>CC1(C)C=Cc2c(ccc3ccc(=O)oc23)O1</smiles>

Seselina

Piranocumarina angular (Pca)

Wikstrosina

Triscumarina (Tc)<smiles>COc1cc2cc(Oc3ccc4ccc(=O)oc4c3)c(=O)oc2cc1O</smiles>

Daphnoretina

Biscumarina $(\mathrm{Bc})$

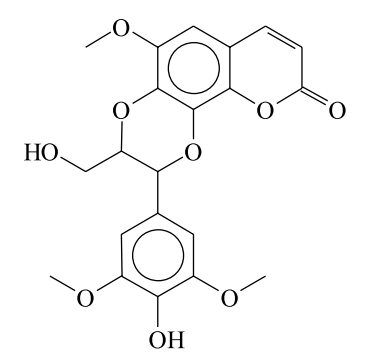

Aquilochina

Figura 1. Tipos de cumarinas

A Figura 2 mostra a ocorrência das cumarinas produzidas por famílias de Angiospermae, sendo que as famílias com NO > 100 foram representadas separadas e as famílias com NO $<100$ tiveram suas ocorrências somadas e o total foi apresentado como Outras.
Tabela 2. Número de ocorrências (NO) e número percentual de ocorrências (NO\%) e número de tipos (NT) para famílias pertencentes às ordens com ocorrência $\geq 20$

\begin{tabular}{|c|c|c|c|c|}
\hline Ordens & Famílias & NO & $\mathrm{NO} \%$ & NT \\
\hline \multirow[t]{2}{*}{ Araliales } & Apiaceae & 3041 & 99.78 & 5 \\
\hline & Araliaceae & 7 & 0.22 & 1 \\
\hline \multirow[t]{5}{*}{ Rutales } & Rutaceae & 1683 & 97.63 & 6 \\
\hline & Meliaceae & 30 & 1.74 & 2 \\
\hline & Simaroubaceae & 9 & 0.56 & 2 \\
\hline & Burseraceae & 1 & 0.06 & 1 \\
\hline & Cneoraceae & 1 & 0.06 & 1 \\
\hline Asterales & Asteraceae & 830 & 100.00 & 2 \\
\hline Oleales & Oleaceae & 149 & 100.00 & 1 \\
\hline \multirow[t]{2}{*}{ Fabales } & Fabaceae & 237 & 96.73 & 5 \\
\hline & Caesalpiniaceae & 8 & 3.27 & 1 \\
\hline \multirow[t]{3}{*}{ Urticales } & Moraceae & 135 & 97.13 & 4 \\
\hline & Urticaceae & 3 & 2.16 & 1 \\
\hline & Ulmaceae & 1 & 0.72 & 1 \\
\hline Thymelaeales & Thymelaeaceae & 121 & 100.00 & 7 \\
\hline \multirow[t]{6}{*}{ Gentianales } & Rubiaceae & 59 & 64.84 & 1 \\
\hline & Apocynaceae & 21 & 23.08 & 3 \\
\hline & Menyanthaceae & 4 & 4.40 & 2 \\
\hline & Gentianaceae & 2 & 2.20 & 1 \\
\hline & Asclepiadaceae & 3 & 3.30 & 1 \\
\hline & Loganiaceae & 2 & 2.20 & 1 \\
\hline \multirow[t]{3}{*}{ Solanales } & Solanaceae & 59 & 62.90 & 1 \\
\hline & Convolvulaceae & 21 & 33.87 & 2 \\
\hline & Polemoniaceae & 2 & 3.23 & 2 \\
\hline Rosales & Rosaceae & 69 & 100.00 & 3 \\
\hline \multirow[t]{2}{*}{ Saxifragales } & Saxifragaceae & 40 & 65.57 & 1 \\
\hline & Crassulaceae & 21 & 34.43 & 1 \\
\hline Euphorbiales & Euphorbiaceae & 47 & 100.00 & 3 \\
\hline \multirow[t]{2}{*}{ Caryophyllales } & Caryophyllaceae & 33 & 71.74 & 1 \\
\hline & Chenopodiaceae & 13 & 28.26 & 2 \\
\hline \multirow[t]{4}{*}{ Sapindales } & Sapindaceae & 18 & 40.91 & 1 \\
\hline & Hippocastanaceae & 21 & 47.73 & 2 \\
\hline & Aceraceae & 4 & 9.09 & 2 \\
\hline & Anacardiaceae & 1 & 2.27 & 1 \\
\hline \multirow{2}{*}{ Lamiales } & Lamiaceae & 23 & 95.83 & 2 \\
\hline & Verbenaceae & 1 & 4.17 & 1 \\
\hline \multirow[t]{5}{*}{ Malvales } & Malvaceae & 10 & 45.46 & 2 \\
\hline & Sterculiaceae & 7 & 31.82 & 1 \\
\hline & Dipterocarpaceae & 2 & 9.10 & 1 \\
\hline & Cistaceae & 2 & 9.10 & 1 \\
\hline & Elaeocarpaceae & 1 & 4.55 & 1 \\
\hline Ericales & Ericaceae & 35 & 100.00 & 1 \\
\hline Ranunculales & Ranunculaceae & 21 & 100.00 & 1 \\
\hline
\end{tabular}

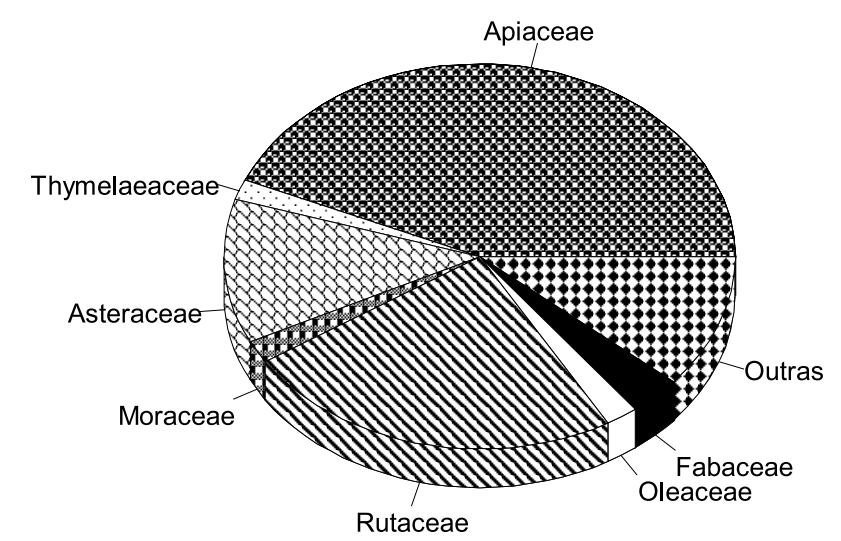

Figura 2. Ocorrência de cumarinas nas principais famílias em Angiospermae produtoras de cumarinas contendo $\mathrm{NO}>100$ 
A Tabela 3 mostra as sete maiores famílias produtoras de cumarinas Apiaceae, Rutaceae, Asteraceae, Fabaceae, Oleaceae, Moraceae e Thymelaeaceae arranjadas na ordem decrescente do número de ocorrências acompanhadas do número percentual de ocorrências dos diferentes tipos cumarínicos que produzem. Fica clara a importância da produção de cumarinas simples em Angiospermae. Além disso, pode-se observar detalhes de especialização na produção desses metabólitos pelas famílias consideradas.

Tabela 3. Número de ocorrência (NO) de cumarinas nas famílias e número de ocorrência percentual $(\mathrm{NO} \%)$ dos tipos de cumarinas produzidas pelas famílias em relação às ordens

\begin{tabular}{|c|c|c|c|}
\hline Ordem & Família & $\mathrm{NO}$ & $\mathrm{NO} \%$ \\
\hline Araliales & Apiaceae & 3041 & $\begin{array}{l}\mathrm{Cs}=32,58 \\
\mathrm{Fcl}=47,54 \\
\mathrm{Fca}=10,53 \\
\mathrm{Pcl}=2,40 \\
\mathrm{Pca}=6,73\end{array}$ \\
\hline Rutales & Rutaceae & 1683 & $\begin{array}{l}\mathrm{Cs}=48,78 \\
\mathrm{Fcl}=33,69 \\
\mathrm{Fca}=1,45 \\
\mathrm{Pcl}=8,53 \\
\mathrm{Pca}=2,26 \\
\mathrm{Bc}=3,02\end{array}$ \\
\hline Asterales & Asteraceae & 830 & $\begin{array}{l}\mathrm{Cs}=98,68 \\
\mathrm{Fcl}=1,33\end{array}$ \\
\hline Fabales & Fabaceae & 237 & $\begin{array}{l}\mathrm{Cs}=77,55 \\
\mathrm{Fcl}=13,70 \\
\mathrm{Fca}=5,71 \\
\mathrm{Pcl}=2,86 \\
\mathrm{Pca}=0,82\end{array}$ \\
\hline Oleales & Oleaceae & 149 & $\mathrm{Cs}=100,00$ \\
\hline Urticales & Moraceae & 135 & $\begin{array}{l}\mathrm{Cs}=14,29 \\
\mathrm{Fcl}=22,19 \\
\mathrm{Fca}=3,04 \\
\mathrm{Pcl}=2,74\end{array}$ \\
\hline Thymelaeales & Thymelaeaceae & 121 & $\begin{array}{l}\mathrm{Cs}=52,89 \\
\mathrm{Fcl}=1,65 \\
\mathrm{Fca}=2,48 \\
\mathrm{Pcl}=1,65 \\
\mathrm{Lc}=2,48 \\
\mathrm{Bc}=36,36 \\
\mathrm{Tc}=2,48\end{array}$ \\
\hline
\end{tabular}

As Figuras 3-9 mostram graficamente os dados apresentados na Tabela 3. Interessante observar que dos oitos tipos de cumarinas descritos anteriormente, alguns destacam-se tanto pela sua distribuição quanto pela proporção em que ocorrem. As cumarinas simples são as mais espalhadas por toda Angiospermae e em Oleaceae e Asteraceae sua ocorrência é de $100 \%$ e $98,68 \%$, respectivamente (Tabela 3). Em relação às famílias nas quais as cumarinas são consideradas marcadores quimiotaxonômicos, por serem bastante diversificadas como em Apiaceae e Rutaceae, o percentual de ocorrência de cumarinas simples não é tão evidenciado, sugerindo a sua transformação para esqueletos mais complexos. Em Apiaceae e Moraceae as

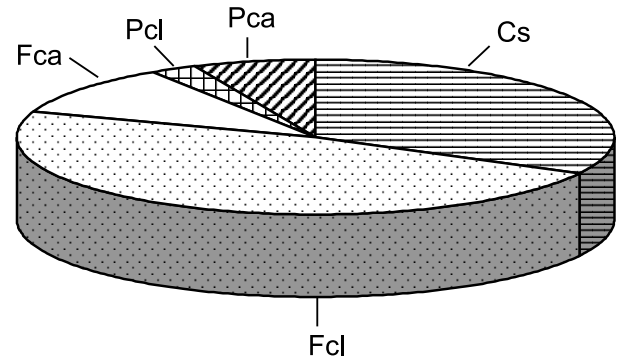

Figura 3. Número percentual de ocorrências de tipos de cumarinas na família Apiaceae em relação à ordem Araliales

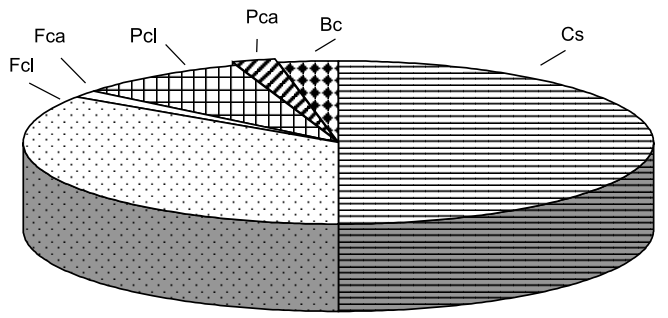

Figura 4. Número percentual de ocorrências de tipos de Cumarinas na família Rutaceae em relação à ordem Rutales

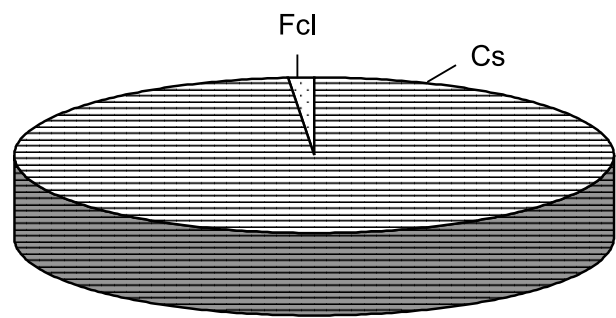

Figura 5. Número percentual de ocorrências de tipos de cumarinas na família Asteraceae em relação à ordem Asterales

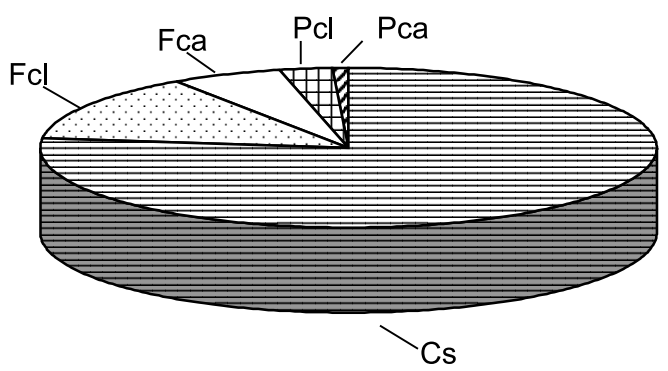

Figura 6. Número percentual de ocorrências de tipos de cumarinas na família Fabaceae em relação à ordem Fabales

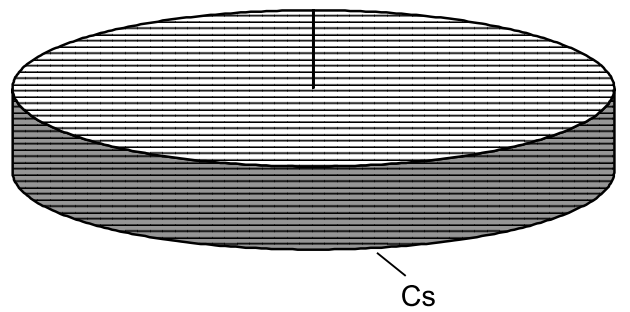

Figura 7. Número percentual de ocorrências de tipos de cumarinas na família Oleaceae em relação à ordem Oleales 


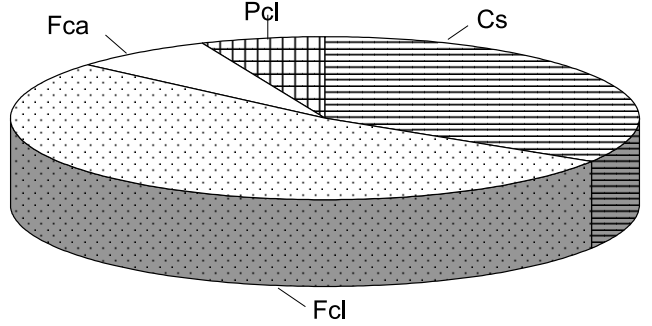

Figura 8. Número percentual de ocorrências de tipos de cumarinas na família Moraceae em relação à ordem Urticales

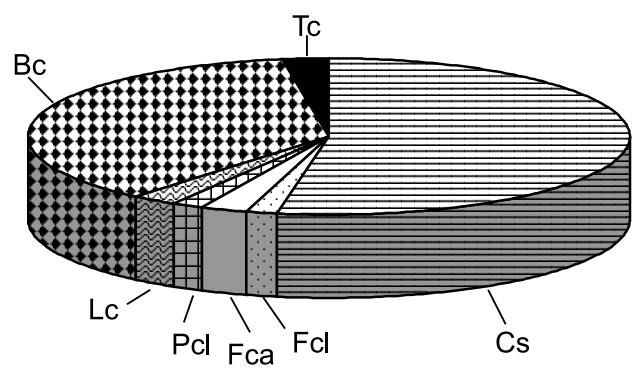

Figura 9. Número percentual de ocorrências de tipos de cumarinas na família Thymelaeaceae em relação à ordem Thymeleales

cumarinas simples aparecem em segundo lugar no que diz respeito ao número percentual de ocorrências. No entanto, em Rutaceae esse tipo cumarínico representa o maior percentual de ocorrências. Outras famílias como Fabaceae, Oleaceae e Thymelaeaceae onde a ocorrência dessa classe de substâncias é bastante significativa, as cumarinas simples são produzidas em maior percentual.

As furanocumarinas lineares são substâncias que ficam mais restritas a algumas famílias. Apresentam ocorrências bastante significativas em Apiaceae e Moraceae onde representam o maior percentual, em Rutaceae e Fabaceae são cumarinas que aparecem em segundo lugar de produção. As furanocumarinas angulares apesar da baixa ocorrência aparecem em Apiaceae, Rutaceae, Fabaceae, Moraceae e Thymelaeaceae.

Um outro tipo de cumarina que se destaca pelo percentual de ocorrência e não pela sua distribuição em famílias de Angiospermae são as bis-cumarinas, presentes significativamente em Thymelaeaceae. Nesse táxon as bis-cumarinas nada mais são do que cumarinas simples ligadas entre si. Observando a distribuição dos tipos cumarínicos nessa família, em nível de número percentual de ocorrências como mostra a Tabela 4, fica bem evidenciado que as furano- e piranocumarinas são bem menos frequentes do que as cumarinas simples, o que reforça análise das cumarinas no táxon em questão. Importante ressaltar que as bis-cumarinas em Rutaceae são formadas por diferentes combinações entre os diversos tipos cumarinas encontrados nesse táxon.

Estudo do número percentual de ocorrências de cumarinas nas famílias de Angiospermae, permitiu a consideração dessas ocorrências em duas maneiras: avaliação da produção de cumarinas simples e avaliação de furano- e piranocumarinas em conjunto. A análise separada das ocorrências das cumarinas simples, trouxe informações bastante interessantes sobre os táxons que mais biossintetizam esse tipo. Na Tabela 4 são apresentados os valores referentes ao Índice de Sporne e ao número percentual de ocorrências tanto para cumarinas simples quanto para o somatório das furano- e piranocumarinas.

A partir das correlações entre NO\% x IS (Figura 10) pode ser
Tabela 4. Índices morfológico e químico referente às famílias produtoras de cumarinas com $\mathrm{NO}>100$

\begin{tabular}{llcr}
\hline Família & IS & NO\% Cs & $\Sigma[\mathrm{A}+\mathrm{B}]$ \\
\hline Apiaceae & 51 & 32,58 & 67,19 \\
Rutaceae & 45 & 48,78 & 45,82 \\
Asteraceae & 72 & 98,68 & 1,00 \\
Fabaceae & 48 & 77,55 & 22,04 \\
Oleaceae & 62 & 100,00 & 0,00 \\
Moraceae & 48 & 14,29 & 27,97 \\
Thymelaeaceae & 55 & 52,89 & 5,80 \\
\hline
\end{tabular}

Legenda: $\mathrm{NO} \% \mathrm{Cs}=$ Número percentual de ocorrências de cumarinas simples.

$\Sigma[\mathrm{A}+\mathrm{B}]=$ Somatório de $(\mathrm{A})=$ número percentual de ocorrências $(\mathrm{NO} \%)$ das furanocumarinas lineares e angulares, somatório de (B) $=$ número percentual de ocorrências $(\mathrm{NO} \%)$ das piranocumarinas lineares e angulares.

observado que em relação as cumarinas simples, as famílias com elevado Índice de Sporne como Oleaceae e Asteraceae são as que apresentam maior número percentual de ocorrências, mostrando dessa maneira uma correlação positiva que indica serem as famílias mais evoluídas, segundo Sporne, as mais especializadas na produção desse tipo de cumarina. Em relação a Asteraceae pode-se dizer que essa família basicamente produz esse tipo de cumarina (cumarinas simples $98.68 \%$ e furanocumarinas lineares $1.33 \%$ ). A Figura 11 mostra os resultados da correlação do número percentual de ocorrências para

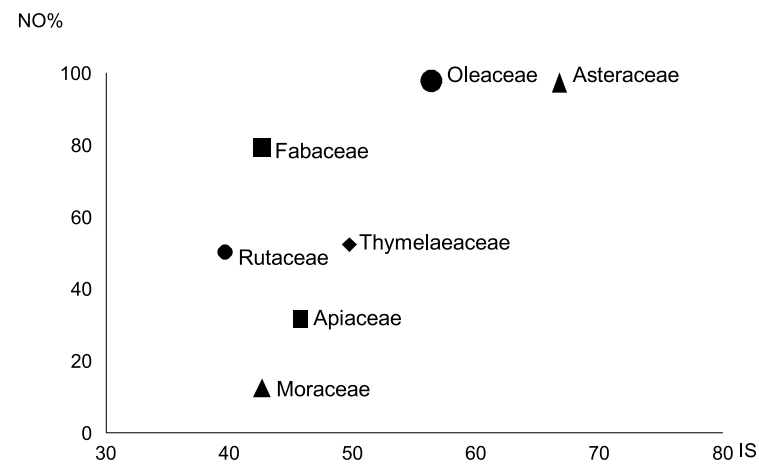

Figura 10. Correlação entre NO\% x IS em relação ao número percentual de ocorrências para cumarinas simples nas famílias produtoras de cumarinas com $N O>100$

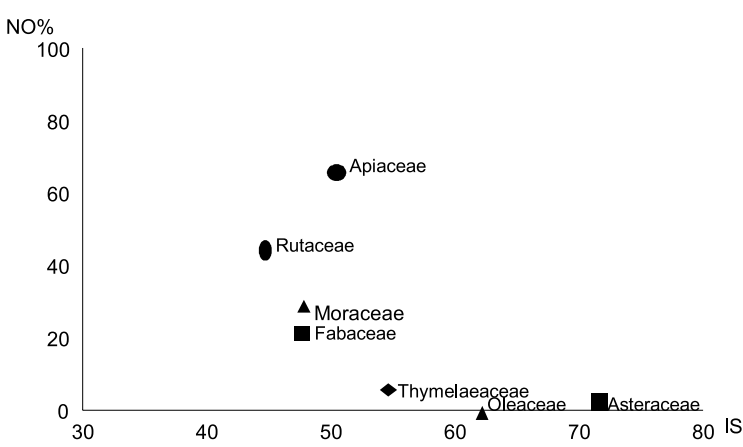

Figura 11. Correlação NO\% x IS do total de furano-e piranocumarinas nas famílias produtoras de cumarinas com NO>100 
furano- e piranocumarinas em conjunto. Interessante observar que as famílias com Índice de Sporne numa faixa entre 40-60 são as que mostram maior ocorrência percentual desses tipos cumarínicos. Asteraceae e Oleaceae, as famílias com maiores Índices de Sporne, são muito pouco ou nada especializadas para a produção desses tipos de cumarinas.

Análise conjunta das Figuras 10 e 11 permite observar uma afinidade, seja na produção de cumarinas simples, seja na produção de furano- e piranocumarinas, entre os grupos: Oleaceae e Asteraceae; Apiaceae e Rutaceae; e Fabaceae e Thymelaeaceae, plenamente concordante com os seus posicionamentos taxonômicos ${ }^{8}$. A família Moraceae, com uma pequena produção de cumarinas simples e um pouco mais especializada em furanocumarinas lineares ocupa posicionamento diferenciado em relação as outras famílias consideradas.

\section{CONCLUSÃO}

A distribuição de cumarinas nas Angiospermae foi verificada em oitenta e sete famílias e conta com a produção preferencial de cumarinas simples. Os demais tipos cumarínicos: furanocumarinas lineares e angulares, piranocumarinas lineares e angulares, lignocumarinas, bis-cumarinas, tris-cumarinas ocorrem mais especificamente em poucas famílias e mostram uma tendência de diversificação nos táxons considerados mais primitivos. A observação de afinidades na produção de cumarinas simples e furano/ piranocumarinas pelas famílias Ole/Ast, Api/Rut e Fab/Thym está de acordo com seus índices de avanço evolutivo morfológico (Índice de Sporne).Apiaceae, Rutaceae, Asteraceae, Fabaceae, Oleaceae, Moraceae e Thymeleaceae são as famílias mais discutidas, visto apresentarem os maiores números de ocorrências (NO>100).

\section{AGRADECIMENTO}

Ao CNPq pelas bolsas concedidas aos autores.

\section{REFERÊNCIAS}

1. Gottlieb, O.R.; Biochem. Syst. Ecol. 1988, 16, 249.

2. Barreiros, E. L.; Tese de Doutorado, Universidade de São Paulo, Brasil, 1990.

3. Soine, T. O.; J. Pharm. Sci. 1964, 53, 231.

4. Gottlieb, O .R.; Borin, M. R. M. B.; Kaplan, M. A . C.; Phytochemistry $\mathbf{1 9 9 5}, 40,99$.

5. Gottlieb, O . R.; Kaplan, M. A. C.; Borin, M. R. M. B.; Biodiversidade Um Enfoque Químico-Biológico, $1^{\mathrm{a}}$ ed., Ed. UFRJ: Rio de Janeiro, 1996.

6. Sporne, K. R.; New Phytol. 1980, 85, 419.

7. Ribeiro, C. V. C. ; Tese de Mestrado, Universidade Federal do Rio de Janeiro, Brasil, 1997.

8. Cronquist, A.; Evolution and Classification of Flowering Plants, $2^{\mathrm{a}}$ ed., New York Botanical Garden, 1988. 